\title{
Tetrasomy 15q26: a distinct syndrome or Shprintzen-Goldberg syndrome phenocopy?
}

\author{
Brynn Levy, MSc (Med), PhD¹, David Tegay, DO², Peter Papenhausen, $\mathrm{PhD}^{3}$, James Tepperberg, $\mathrm{PhD}^{3}$, \\ Odelia Nahum, MS1, Tammy Tsuchida, MD4, Beth A. Pletcher, MD5${ }^{5}$, Leena Ala-Kokko, MD, PhD ${ }^{6}$, \\ Stuart Baker ${ }^{6}$, Barbara Frederick, BA ${ }^{7}$, Kurt Hirschhorn, $\mathrm{MD}^{8}$, Peter Warburton, $\mathrm{PhD}^{8}$ and \\ Alan Shanske, MD ${ }^{9}$
}

\begin{abstract}
Purpose: The aim of this study was to characterize the clinical phenotype of patients with tetrasomy of the distal $15 \mathrm{q}$ chromosome in the form of a neocentric marker chromosome and to evaluate whether the phenotype represents a new clinical syndrome or is a phenocopy of Shprintzen-Goldberg syndrome.

Methods: We carried out comprehensive clinical evaluation of four patients who were identified with a supernumerary marker chromosome. The marker chromosome was characterized by G-banding, fluorescence in situ hybridization, single nucleotide polymorphism oligonucleotide microarray analysis, and immunofluorescence with antibodies to centromere protein C.
\end{abstract}

Results: The marker chromosomes were categorized as being neocentric with all showing tetrasomy for regions distal to $15 \mathrm{q} 25$ and the common region of overlap being $15 \mathrm{q} 26 \rightarrow$ qter.

Conclusion: Tetrasomy of $15 \mathrm{q} 26$ likely results in a distinct syndrome as the patients with tetrasomy $15 \mathrm{q} 26$ share a strikingly more consistent phenotype than do the patients with Shprintzen-Goldberg syndrome, who show remarkable clinical variation.

Genet Med 2012:14(9):811-818

Key Words: craniosynostosis; Marfanoid habitus; neocentromere; Shprintzen-Goldberg syndrome; tetrasomy 15q26

\section{INTRODUCTION}

The hallmarks of Shprintzen-Goldberg syndrome (SGS) are craniosynostosis and Marfanoid habitus. The first unambiguous case of SGS was reported by Sugarman and Vogel in $1981 .{ }^{1}$ Shprintzen and Goldberg described two unrelated patients with the disorder now known as SGS a year later. ${ }^{2}$ There have been more than 40 cases reported in the literature. Additional clinical features, which are present in at least two-thirds of the cases, include scaphocephaly, hypertelorism, exophthalmos, malformed pinnae, micrognathia, high-arched palate, pectus excavatum, arachnodactyly, developmental delay, and hypotonia. ${ }^{3}$ The etiology of SGS is uncertain but has been linked to mutations in the fibrillin-1 gene (15q21.1 $)^{4,5}$ as well as TGFBR1 (9q22) and TGFBR2 (3p22). ${ }^{3,4,6,7}$ Mutations in TGFBR1 and TGFBR2 have also been identified in individuals with LoeysDietz syndrome, which is often difficult to distinguish clinically from SGS. ${ }^{6-9}$

Patients with supernumerary marker chromosomes can present with a wide array of phenotypes, ranging from normal to severely affected, depending on the degree and the type of aneuploidy. Some rare supernumerary chromosomes contain a centromeric constriction, but no a satellite DNA, which is found at all endogenous centromeres. These chromosomes have acquired neocentromeres, ectopic centromeres that have epigenetically formed on single-copy DNA, and provide mitotic stability to supernumerary chromosomes that would otherwise be acentric and lost. The majority of neocentromeres are found on inversion duplication chromosomes, which result in partial tetrasomy or trisomy. ${ }^{10-13}$

We report four cases of partial tetrasomy of the distal segment of the long arm of chromosome 15, all in the form of an inverted duplicated supernumerary chromosome with a neocentromere. All patients have a distinctive phenotype that is consistent with previously reported cases of distal tetrasomy $15 \mathrm{q}$ resulting from a supernumerary marker chromosome. Although patients with distal tetrasomy $15 \mathrm{q}$ show some of the features present in patients with the SGS, their overall phenotypic gestalt appears to be different.

\section{MATERIALS AND METHODS}

\section{Patients}

Informed consent was obtained for reporting the clinical spectrum of all patients as well as for public release of patient photographs.

\footnotetext{
${ }^{1}$ Department of Pathology and Cell Biology, Columbia University Medical Center and the New York Presbyterian Hospital, New York, New York, USA; ${ }^{2}$ Department of Medicine, New York College of Osteopathic Medicine, Old Westbury, New York, USA; ${ }^{3}$ Center for Molecular Biology and Pathology, LabCorp, Research Triangle Park, Durham, North Carolina, USA; ${ }^{4}$ Department of Neurology, Children's National Medical Center, Washington, DC, USA; ${ }^{5}$ Department of Pediatrics, University of Medicine and Dentistry of New Jersey, Newark, New Jersey, USA; ${ }^{6}$ Connective Tissue Gene Tests, Allentown, Pennsylvania, USA; ${ }^{7}$ Coriell Institute for Medical Research, Camden, New Jersey, USA; ${ }^{8}$ Department of Human Genetics, Mount Sinai School of Medicine, New York, New York, USA; ${ }^{9}$ Center for Craniofacial Disorders, Children's Hospital at Montiefiore, Bronx, New York, USA.

Correspondence: Brynn Levy (BL2185@Columbia.edu)
} 
Patient 1. The proband (Figure 1a) was the 3,239-g (25\%) product of a full-term uneventful pregnancy, delivered by normal spontaneous vaginal delivery to a 30 -year-old gravida 9 para 6026 mother. During the neonatal period, he had mild respiratory distress requiring continuous positive airway pressure, as well as feeding difficulties. At the age of 6 years and 9 months, he weighed $17 \mathrm{~kg}(<3 \%)$, his length was $111 \mathrm{~cm}(5 \%)$, and his head circumference $(\mathrm{HC})$ was $53 \mathrm{~cm}(75 \%)$, and he was noted to have significant postnatal growth retardation, macrocephaly, dolicocephaly, turricephaly, an elongated face, hypertelorism, flat philtrum, posteriorly rotated pinnae, a broad nasal root, and an extremely high vaulted and narrow palate with a bifid uvula. He had severe kyphoscoliosis and an asymmetric thorax with a prominent tail-like coccyx. His extremities were long and thin with fifth-finger clinodactyly, flexion contractures of the digits, and long overlapping toes (Figure 1a insets). When last seen at 10 years and 3 months, he weighed $26.7 \mathrm{~kg}$ (10\%), his height was $130 \mathrm{~cm}(5 \%)$, and his HC was $55 \mathrm{~cm}(95 \%)$ ). A renal ultrasound showed normal sized kidneys with mild rotation and pelviectasis of the right kidney. A computerized axial tomography scan of the head revealed hydrocephalus and rostral agenesis of the corpus callosum as well as atrophy of the falx cerebri. He was nonverbal and severely developmentally delayed with a mild left-sided hearing loss but was able to communicate his needs with gestures. Molecular analysis of three genes (FBN1, TGFBR1/2) associated with Marfan-like connective tissue disorders including SGS was normal.

Patient 2. The proband (Figure 1b) was the 3,875-g $(75 \%$ ) product of a term pregnancy delivered by cesarean section because of a breech presentation to a 28 -year-old primigravida. The birth length and $\mathrm{HC}$ were unavailable. She required strabismus repair and stent placement for an obstructive uropathy within the first year and scoliosis surgery when she was older. She developed seizures and is currently on Paxil and Lamictal. Her physical examination at the age of 19 years revealed a tall dysmorphic young woman with a height of $168 \mathrm{~cm}(75 \%)$ and a $\mathrm{HC}$ of 55 $\mathrm{cm}(50 \%)$. She had turricephaly, a long face, hypertelorism, a downward obliquity of the palpebral fissures, protuberant nose, high narrow palate, and dental crowding. Skeletal abnormalities included scoliosis, arachnodactyly, and camptodactyly of digits 2, 3 , and 4 on the right and of the distal interphalangeal joint on the left third digit. She was severely developmentally delayed with bilateral hearing loss (right worse than left). She communicates with gestures and signs and has a history of self-mutilation.

Patient 3. The proband (Figure 1c) was the 2,317-g (10\%o) product of a 37 week in vitro fertilization pregnancy delivered to a 37-year-old gravida 5, para 1031 mother. The in vitro fertilization triplet pregnancy was reduced spontaneously to a singleton. Amniocentesis revealed mosaicism for a small marker chromosome. At birth, she was $47 \mathrm{~cm}$ long (10\%) with a $\mathrm{HC}$ of $32 \mathrm{~cm}(2 \%)$. She had a dysmorphic skull and was noted to have a horseshoe kidney with hydronephrosis, and metopic and bilateral coronal craniosynostoses. She underwent a craniotomy at 5 months of age. When first seen at 4.5 years, her physical examination revealed a dysmorphic child with turricephaly, high-arched palate, thoracodorsal scoliosis, arachnodactyly, absence of digital flexion creases, contractures of fingers 2, 3, and 4 bilaterally, and clinodactyly of both fifth a

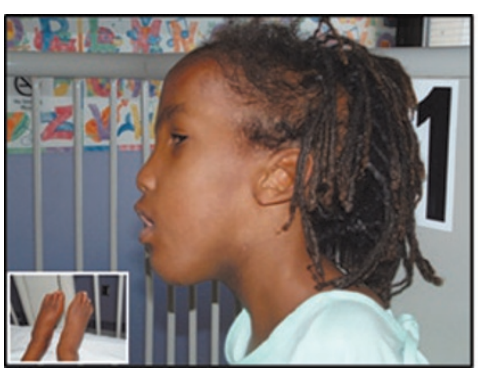

c

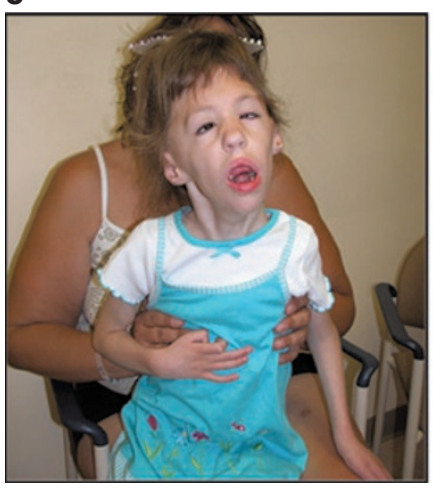

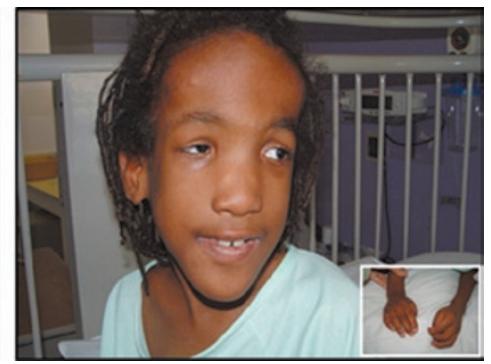

b
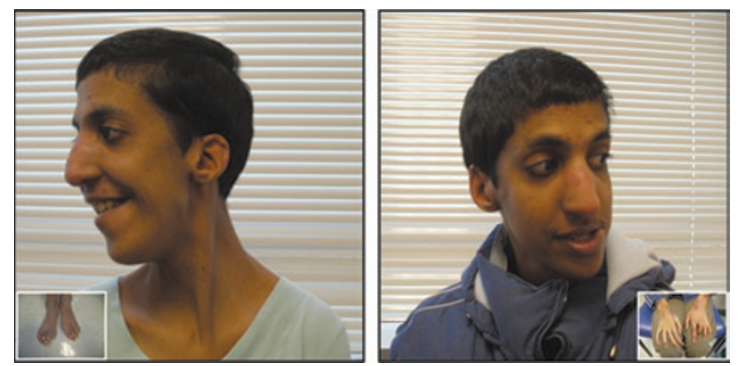

d

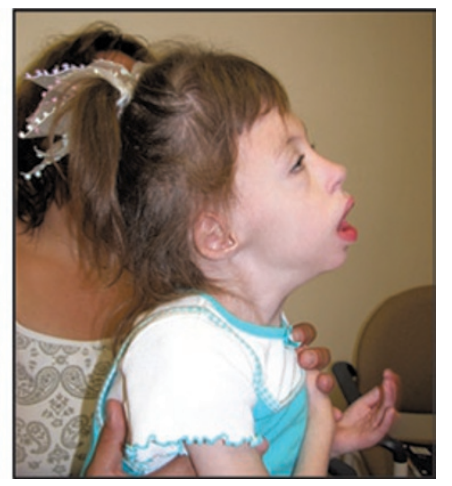

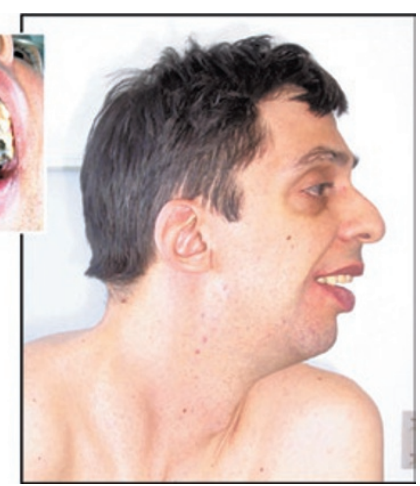

Figure 1 Observable phenotypic features. (a) Patient 1 at age 6. (b) Patient 2 at age 19. (c) Patient 3 at age $41 / 2$. (d) Patient 4 at age 32. 

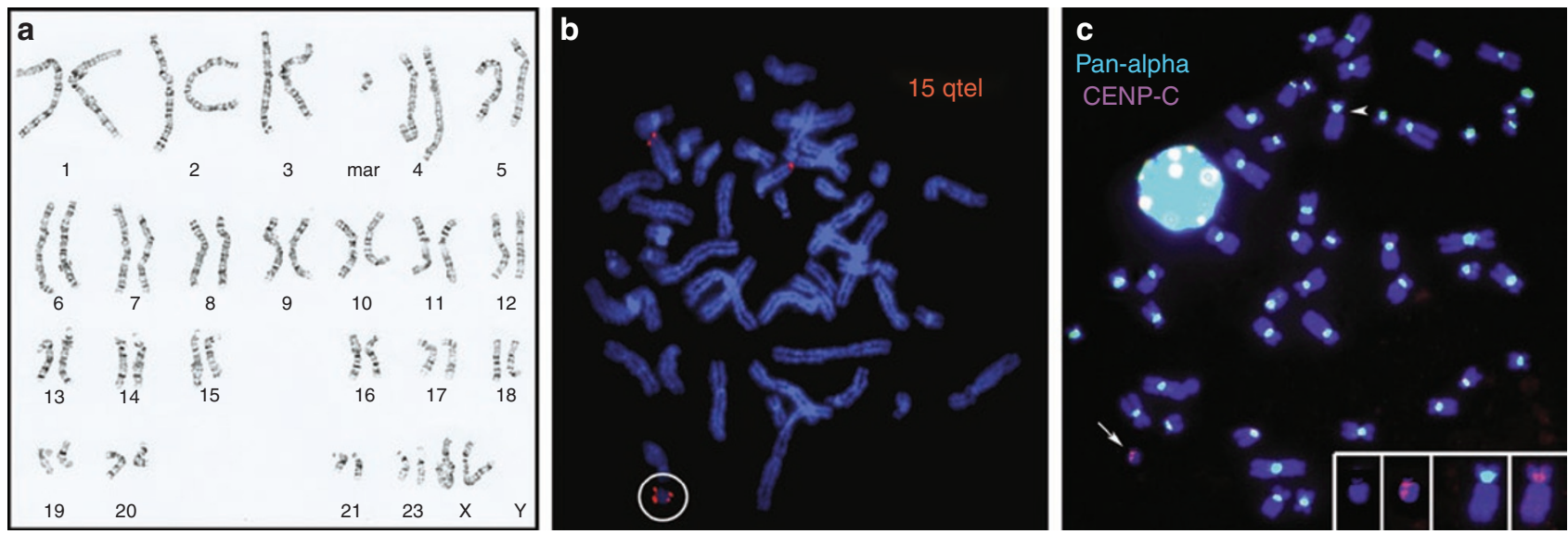

Figure 2 Molecular and cytogenetic workup. (a) GTG metaphase showing the marker in patient 3. (b) Metaphase FISH with 15q subtelomeric probe in patient 4 showing normal hybridization patterns on the two normal chromosomes 15 and two distinct pairs of signals at each end of the marker chromosome (white circle). (c) Immuno-FISH analysis of neocentromere. FISH was performed with a pan- $\alpha$ all centromere probe (Cytocell, Cambridge, UK), which hybridized to all normal centromeres (green) (e.g., white arrowhead and inset) but not to the neocentromere (white arrow and inset). Immunofluorescence with antibodies to CENP-C is seen at all centromeres (red) including the neocentromere (white arrow and inset) and normal centromeres (inset). CENP-C, centromere protein C; FISH, fluorescence in situ hybridization.

fingers. A syrinx at the thoracic level was seen on magnetic resonance imaging. She was nonverbal and severely developmentally delayed. Molecular analysis of three genes ( $F B N 1$, TGFBR1/2) associated with Marfan-like connective tissue disorders including SGS was normal.

Patient 4. The proband (Figure 1d) was first evaluated at 32 years of age for overgrowth, Marfanoid habitus and mild mental retardation. He was delivered full term via C-section to a 25-year-old gravida 2 para 1001 mother; he had a birthweight of 5,900 g and length of $63 \mathrm{~cm}$ (both $>97 \%$ ). He underwent surgery for craniosynostosis at 8 months of age and for a 70-degree thoracic scoliosis at 29 years of age. Global developmental delay was present with a full-scale Wechsler Adult Intelligence Scale Interpretation IQ of 57. At 28 years of age, computerized axial tomography scan of the head detected mild cortical atrophy; and a similar scan of the abdominal and pelvic areas was unremarkable and a transthoracic echocardiogram revealed mild mitral regurgitation, tricuspid regurgitation, and pulmonary arterial hypertension with no aortic root enlargement. At 32 years of age, his height was $193.5 \mathrm{~cm}(>97 \%$ ) and his $\mathrm{HC}$ was $57 \mathrm{~cm}(75 \%)$. He was dysmorphic with dolicocephaly, malar flattening, downward obliquity of the palpebral fissures, low-set pinnae, a pseudo-cleft palate, retrognathia, pectus excavatum, and large hands. Molecular analysis of three genes (FBN1, TGFBR1/2) associated with Marfan-like connective tissue disorders including SGS was normal.

\section{Molecular cytogenetic analysis}

Fluorescence in situ hybridization using a 15q subtelomeric probe (Abbott Diagnostics, Abbott Park, IL) and a biotinylated all-human-centromere ( $\alpha$ satellite) (Oncor, Gaithersburg, MD) was performed according to the directions of the manufacturer. Digital image analysis was performed using a Cytovision system (Applied Imaging, Santa Clara, CA).

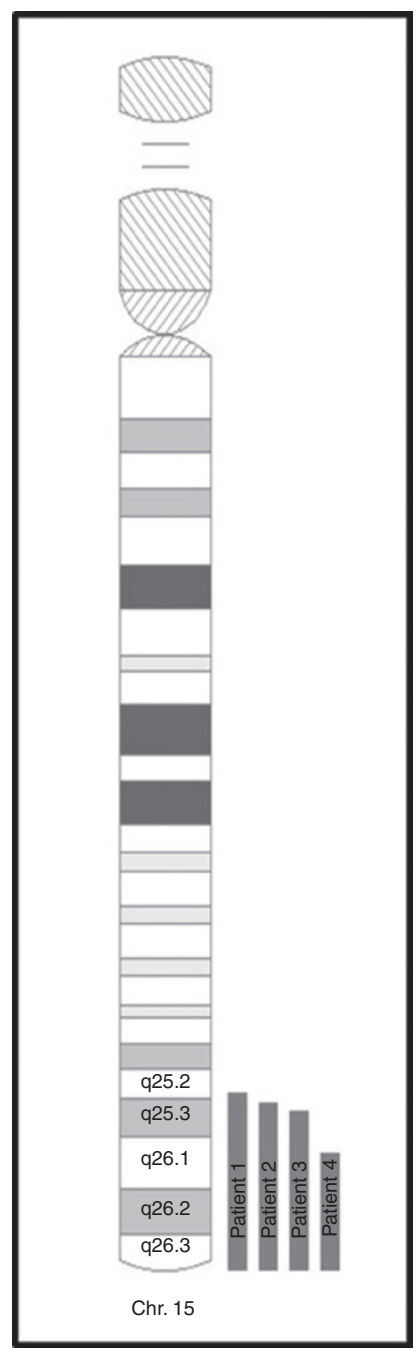

Figure 3 Chromosomal origins of the neocentric marker chromosomes as determined by SOMA. SOMA, single-nucleotide polymorphism oligonucleotide microarray analysis. 
Immunofluorescence with antibodies to CENP-C was performed on 3:1 methanol:acetic acid-fixed chromosomes from primary lymphocytes. Rabbit anti-CENP-C was detected with goat anti-rabbit IgG. Chromosomes were counterstained with 4,6-diamidino-2-phenylindole (DAPI). Photomicrographs were obtained on a Nikon Eclipse 800 (Nikon, Melville, NY) fluorescent/phase microscope equipped with a Sony DKC 5000 (San Diego, CA) camera. Each image was collected separately using single-pass filter sets (Chroma Technology, Bellows Falls, VT), and separate red green blue color channels were merged using Adobe Photoshop (Adobe Systems, San Jose, CA).

Single-nucleotide polymorphism (SNP) oligonucleotide microarray analysis (SOMA) was performed using the Affymetrix Genome Wide Human SNP Array 6.0 (Affymetrix, Santa Clara, CA), which includes more than 906,600 SNPs and more than 946,000 probes for the detection of copy-number variation. Sample preparation, hybridization, and scanning were performed using GeneChip Instrument System hardware according to the specifications of the manufacturer (Affymetrix, Santa Clara, CA). Analysis was performed using the Affymetrix Genotyping Console software. The samples met Affymetrix recommended values for Contrast quality control (SNP) and Median of the Absolute values of all Pairwise Differences quality control (copy number variation)). The intensities of both SNP and copy number variation probes were used to determine segments that varied in copy number. SNP and copy number intensities were normalized against an internally derived normal reference set of 48 samples.

\section{RESULTS}

\section{Conventional and molecular cytogenetic studies}

Peripheral blood chromosome analysis on all four patients revealed a small nonmosaic supernumerary marker chromosome (Figure 2a). SOMA was performed on all four patients and revealed a gain consistent with four copies of the distal region of chromosome 15 . The breakpoints in cases 1-4 were $15 q 25.2 \rightarrow q 26.3(17.3 \mathrm{Mb}), 15 q 25.3 \rightarrow q 26.3(16.6 \mathrm{Mb})$, $15 \mathrm{q} 25.3 \rightarrow \mathrm{q} 26.3(16.1 \mathrm{Mb})$, and $15 \mathrm{q} 26.1 \rightarrow \mathrm{q} 26.3(11.4 \mathrm{Mb})$, respectively (Figure 3 ). Fluorescence in situ hybridization. using a $15 \mathrm{q}$ subtelomeric probe revealed two distinct pairs of signals at each end of the marker, consistent with an inversion duplication of the distal $15 q$ sequences (Figure $2 \mathbf{b}$ ). The $15 q$ probe also hybridized to the telomeric region of each normal chromosome 15, confirming partial tetrasomy for 15q (Figure 2b). This genomic region is rich in duplications and structural variation that may have played a role in the formation of these inverted duplication chromosomes (Figure 4). The absence of a satellite DNA on the marker was confirmed by using an "allhuman-centromere" pan-alpha satellite probe, which hybridized to all normal human centromeres but did not hybridize to the marker chromosome (Figure $2 \mathrm{c}$ ). The presence of a functional centromere on the marker chromosome was confirmed by immunofluorescence with antibodies to CENP-C, which showed the characteristic double-dot CENP-C kinetochore pattern at the centromeres of all chromosomes including the marker (Figure 2c). The clinical features of patients 1-4 are shown in Table 1 together with previously reported cases of chr15: $\quad 82500000$ $15 q 25.2$
83000000
84000000

Breakpoints

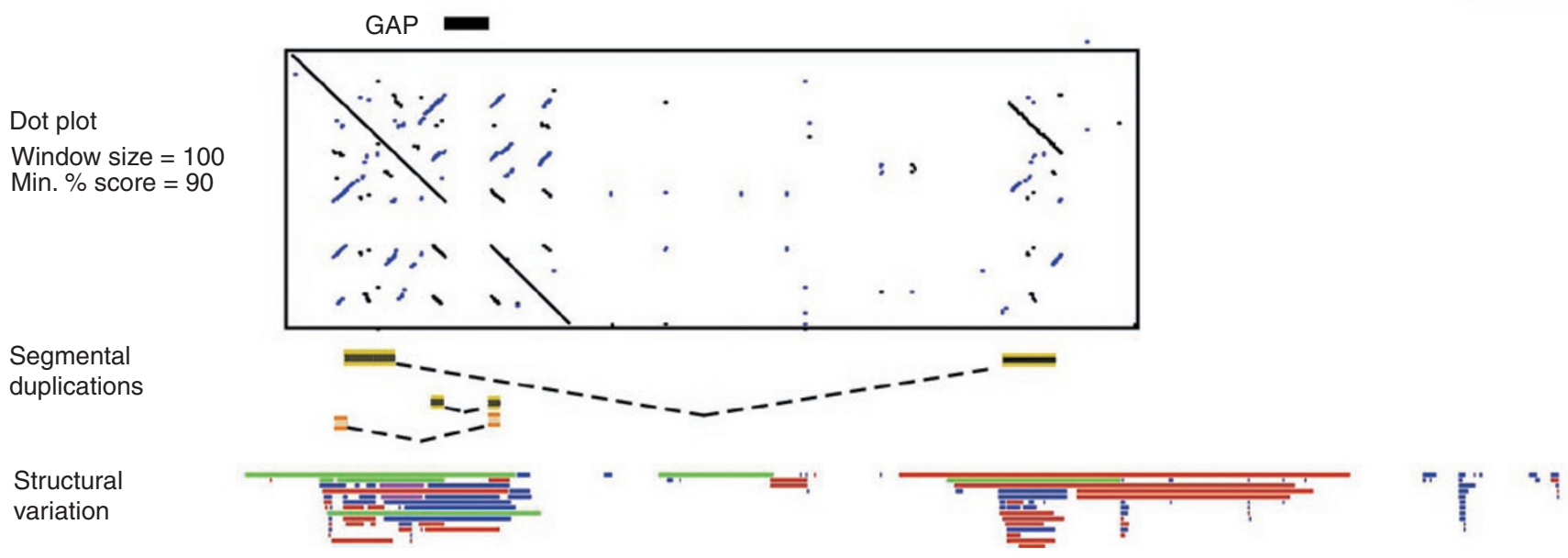

Figure 4 Significant structural variation and segmental duplication at location of inversion duplication breakpoints. A 2-Mbp region of chromosome 15 (UCSC hg18 genome browser) surrounding three proximal breakpoints. The most proximal breakpoint falls just distal to a region with significant structural variation and duplications surrounding a gap, as seen on the dotplot and segmental duplications track (only segmental duplications from the region that are $>98 \%$ homologous are shown). The next breakpoint falls just distal to another region with homology to the first region. Both regions show large amounts of structural variation as seen on the structural variation track (UCSC genome browser). DGV, database of genomic variants; UCSC, University of California, Santa Cruz. 


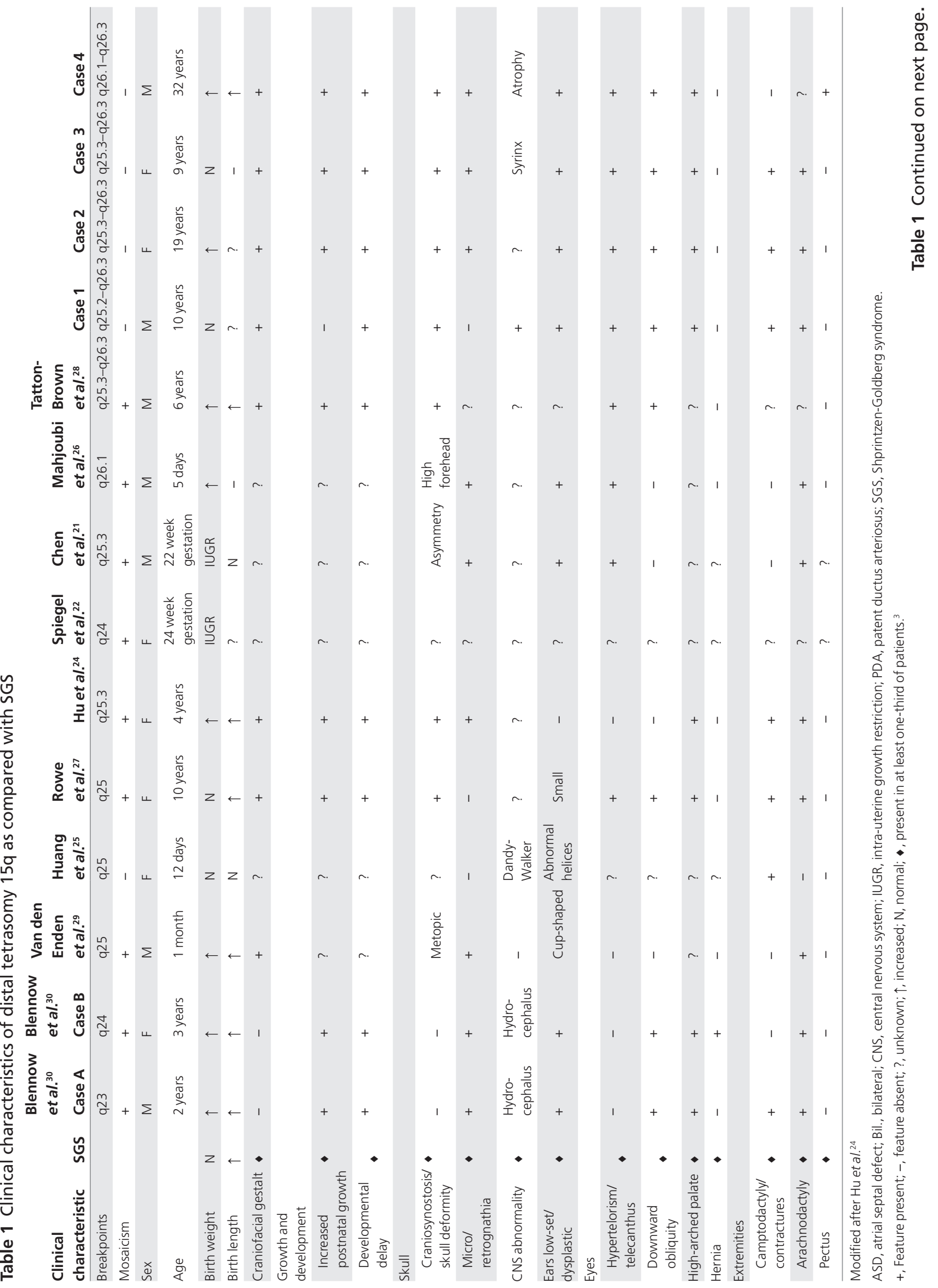




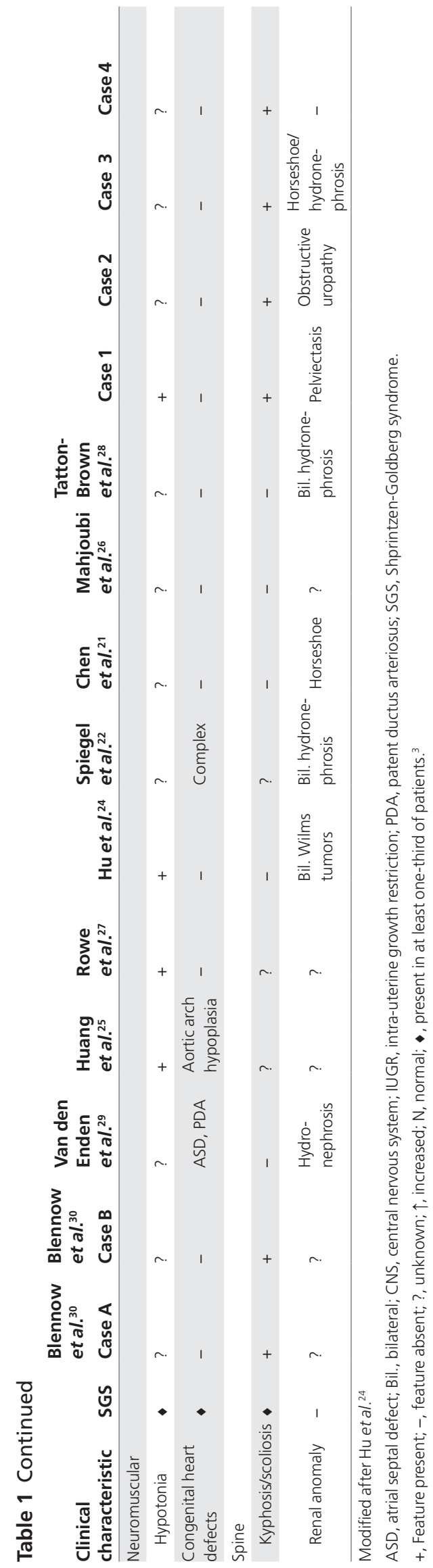

distal tetrasomy 15q. A summary of the molecular cytogenetic results indicating the physical basepair positions on the human genome map (human genome build 36) is shown in Table 2.

\section{DISCUSSION}

Neocentromeres are found on supernumerary chromosomes that have separated from endogenous centromeres, leading to the mitotic stability of paracentric ring chromosomes or distal inversion duplications. Thus, the formation of neocentromeres, while extremely rare, permits aneuploidy for almost any region of the genome, albeit an ascertainment bias for recognized pregnancy or fetal survival. ${ }^{14}$ The inversion duplication $15 \mathrm{q}$ chromosome described in this report ranks as one of the most commonly observed neocentric chromosomes. This hotspot of neocentromere emergence in 15q24-q26 has been attributed to the possible reactivation of an ancestral latent centromere in band 15q25.2 that was inactivated about 25 million years ago. ${ }^{15,16}$

Chromosome $15 \mathrm{q} 24-\mathrm{q} 26$ is rich in segmental duplications and structural variation, which may promote chromosome rearrangements in this area. ${ }^{17}$ These segmental duplications may be pathogenic, and rearrangements of them have been associated with susceptibility for panic and phobic disorders and joint laxity. ${ }^{18}$ Genomic analysis shows that just proximal to the breakpoints in our cases (Figure 4) are two clusters of segmental duplications that contain a gap, inverted repeats surrounding this gap, structural variation, and homology to the more distal cluster. This high degree of structural variation in the region of the breakpoints including inverted repeats suggest that non-allelic homologous recombination between these homologous regions may have played a role in the generation of these inverted duplications. ${ }^{19}$ The evolutionary history of this region is thus related to a high frequency of neocentromere emergence and pathogenic rearrangements.

Distal tetrasomy $15 \mathrm{q}$ is a rare disorder for which molecular cytogenetic techniques such as high-resolution SOMA are useful in delineating the nature of the chromosomal abnormality. There have been at least 13 previously reported cases of tetrasomy $15 q$ in the form of a marker chromosome. ${ }^{10,11,15,20-29}$ However, in only 10 of the 13 are specific clinical data available (Table 1) with 9 of the 10 reported as mosaic tetrasomy 15q. Nonetheless, the craniofacial gestalt observed in all our cases is also present in eight of eight cases tetrasomic for 15q25 where clinical images are available (Table 1). Renal anomaly is also a common feature among distal tetrasomy $15 q$ patients but may not necessarily be reported if not specifically investigated. It is also possible that the critical gene/s responsible for the renal anomalies are located between the genomic coordinates $84,151,101(15 \mathrm{q} 25.3)$ and $88,847,491$ (15q26.1), as suggested by the breakpoint defining the common region of overlap in the three cases with renal anomalies and the starting breakpoint in the case that did not present with renal anomalies.

The molecular etiology of the SGS is not well defined, but a few case reports have linked the phenotype to mutations in three genomic loci, FBN1 at 15q21.1, ${ }^{4,5}$ TGFBR1 at 9q22, ${ }^{3}$ and TGFBR2 at $3 \mathrm{p} 22 .{ }^{4}$ This suggests that there are multiple 
Table 2 Summary of the SOMA results

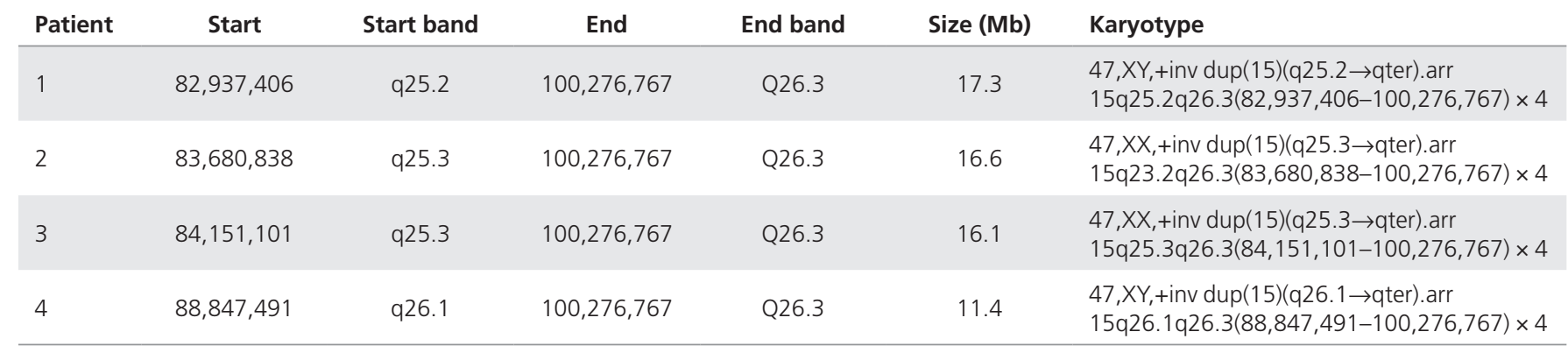

inv dup, inversion duplication; SOMA, single-nucleotide polymorphism oligonucleotide microarray analysis.

genetic factors that result in a common clinical phenotype. The seemingly common craniofacial and skeletal anomalies in our patients raise the question whether the $15 \mathrm{q} 26 \rightarrow \mathrm{qter}$ region is implicated as a fourth candidate region in the etiology of SGS. According to some authors, the SGS phenotype can be subdivided into patients with and without aortic dilatation. ${ }^{8,30}$ Megarbane and Hokayem ${ }^{31}$ further describe type I SGS patients with craniosynostosis and Marfanoid habitus and type II SGS patients with normal intelligence, aortic root anomalies, and mild skeletal dysplasia. ${ }^{31}$ Our patients have features of the SGS phenotype without aortic manifestations and have craniosynostosis with a Marfanoid habitus. In addition, renal anomalies, which are not reported in SGS, were present in three of the four of our patients. The facial dysmorphism in our tetrasomy $15 \mathrm{q} 26$ patients appears different from the facial gestalt presented in the original 1982 Sphrinzen and Goldberg article. ${ }^{2}$ The relatively coarse asymmetric facies, prominent nose with a broad nasal root, and prominent chin are remarkable features in our cohort of patients but not in SGS patients. SGS patients present with mild to moderate delays and are usually verbal. In contrast, our patients are severely developmentally delayed and three of the four patients were nonverbal. Two of the four patients in our cohort had hearing loss, which has not been reported in SGS. Furthermore, the shallow orbits, which are commonly observed in SGS, are not present in our patients.

Our four patients display well-defined clinical features that are remarkably similar to previously reported cases of tetrasomy $15 \mathrm{q}$ involving the most distal region. The distinct phenotypic similarity among the tetrasomy $15 \mathrm{q}$ cases indicates that this is a discrete clinical entity. It is obvious that the $15 \mathrm{q} 25-\mathrm{q} 26$ region harbors dosage-sensitive genes and trisomy for this region also manifests in a spectrum of overlapping anomalies including overgrowth, learning difficulties, renal anomalies, and dysmorphic facies. ${ }^{27,32,33}$ The facies in some trisomy reports appear identical to those of our patients $s^{33}$ and those in other reports appear distinctly different. ${ }^{27,32}$ The dosage threshold required to consistently produce the phenotype described in this report appears to be reached once four copies of the distal 15q region are present. Aneusomy of 15q25-q26 clearly leads to a range of recognizable clinical anomalies and tetrasomy of this region may represent the most severe side of the phenotype. The common region of overlap in our four cases is q26.1-q26.3 (genomic coordinates:
$88,847,491-100,276,767 ; 11.4 \mathrm{Mb}$ ), which implies that this may be the critical region in the tetrasomy $15 \mathrm{q}$ syndrome. Because many of the trisomic cases were defined only by standard cytogenetic G-band analysis and not by high-resolution molecular techniques such as SOMA, it is possible that those reported trisomy cases without the phenotypic gestalt of our four cases did not have aneusomy of the critical 15q26.1-q26.3 region. An alternative hypothesis, explaining the consistent phenotype in tetrasomy $15 \mathrm{q} 26.1-\mathrm{q} 26.3$ as compared with trisomy of the same region, implicates epigenesis. It is felt that neocentromere activity is acquired epigenetically, not as the result of a DNA sequence change. It is possible that part of the phenotype in these neocentric cases is also due to epigenetic effects.

There are more than 60 genes located in the critical 15q26.1q26.3 region and many have yet to be characterized. The various clinical features observed in the tetrasomy $15 q$ phenotype are likely caused by the cascading effects of gene-dosage increases of various genes. Among the candidates are UNC45A, which has cytoskeletal and muscle-specific functions,${ }^{34}$ and IQGAPI, which is a multidomain scaffold protein that binds to a wide variety of targets and modulates several cellular activities, including cell-cell adhesion, transcription, cytoskeletal architecture, and signaling pathways. ${ }^{35}$ FURIN, located at 15q26.1, cleaves pro-TGFB1 to produce biologically active TGFB1, ${ }^{36}$ and its dysregulation could possibly affect the TGFB1 receptor, which would potentially explain the link between the overlapping features seen in SGS and Loeys-Dietz syndrome. IGF1R, located at 15q26.3, encodes an important component of the insulin-like growth factor axis and is critical to pre- and postnatal growth. It encodes an $\alpha 2 \beta 2$-heterotetrameric protein with ligandstimulated tyrosine kinase activity. The binding of IGF-I to its receptor induces receptor autophosphorylation in the intracellular kinase domain of the $\beta$-subunit and results in activation of the intrinsic tyrosine kinase activity of the IGF1R. Okubo et al. ${ }^{37}$ demonstrated increased rates of cell proliferation and autophosphorylation of IGF1R in skin fibroblasts from a patient with duplication of IGFR1 arising from a parental pericentric inversion. Gene dosage for IGF1R is increased in our patients and may result in increased binding of IGFs and overgrowth. In addition, Cunningham et al. recently reported an association between isolated craniosynostosis and nonsynonymous IGF1R mutations, postulating a gain-of-function mechanism. ${ }^{38}$ 
The striking phenotypic resemblance of our four cases certainly points to a role of genes in 15q26 in the etiology of this unique syndrome. The hallmark features include severe developmental delay, craniosynostosis, Marfanoid habitus, coarse asymmetric facies, prominent nose with broad nasal root, and prominent chin. Renal anomalies strongly implicate aneusomy of distal 15q, but may be absent if the breakpoint lies distal to 15q25.3. The clinical similarity to SGS is nonetheless evident and because SGS is difficult to distinguish clinically from Loeys-Dietz syndrome, all three syndromes should be considered in the differential diagnosis. Given the inconclusive link between SGS and mutations in FBN1, TGFBR1, and TGFBR2, ${ }^{9}$ conventional cytogenetic analysis should be considered as the first line of testing before expensive sequencing of the putative SGS genes.

\section{DISCLOSURE}

The authors declare no conflict of interest.

\section{REFERENCES}

1. Sugarman G, Vogel M. Craniofacial and musculoskeletal abnormalities. A questionable connective tissue disease. Case report 76. Synd Iden 1981;1:16-17.

2. Shprintzen RJ, Goldberg RB. A recurrent pattern syndrome of craniosynostosis associated with arachnodactyly and abdominal hernias. J Craniofac Genet Dev Biol 1982;2:65-74.

3. Robinson PN, Neumann LM, Demuth $\mathrm{S}$, et al. Shprintzen-Goldberg syndrome: fourteen new patients and a clinical analysis. Am J Med Genet A 2005;135:251-262.

4. Kosaki K, Takahashi D, Udaka T, et al. Molecular pathology of ShprintzenGoldberg syndrome. Am J Med Genet A 2006;140:104-8; author reply 109.

5. Sood S, Eldadah ZA, Krause WL, McIntosh I, Dietz HC. Mutation in fibrillin-1 and the Marfanoid-craniosynostosis (Shprintzen-Goldberg) syndrome. Nat Genet 1996;12:209-211.

6. Stheneur C, Collod-Béroud G, Faivre L, et al. Identification of 23 TGFBR2 and 6 TGFBR1 gene mutations and genotype-phenotype investigations in 457 patients with Marfan syndrome type I and II, Loeys-Dietz syndrome and related disorders. Hum Mutat 2008;29:E284-E295.

7. Loeys BL, Chen J, Neptune ER, et al. A syndrome of altered cardiovascular, craniofacial, neurocognitive and skeletal development caused by mutations in TGFBR1 or TGFBR2. Nat Genet 2005;37:275-281.

8. Adès LC, Morris LL, Power RG, et al. Distinct skeletal abnormalities in four girls with Shprintzen-Goldberg syndrome. Am J Med Genet 1995;57:565-572.

9. Greally MT. Shprintzen-Goldberg Syndrome. GeneReviews at GeneTests. November 2010. <http://www.genetests.org>.

10. Marshall OJ, Chueh AC, Wong LH, Choo KH. Neocentromeres: new insights into centromere structure, disease development, and karyotype evolution. Am J Hum Genet 2008;82:261-282.

11. Liehr T, Utine GE, Trautmann U, et al. Neocentric small supernumerary marker chromosomes (SSMC)-three more cases and review of the literature. Cytogenet Genome Res 2007;118:31-37.

12. Warburton PE. Chromosomal dynamics of human neocentromere formation. Chromosome Res 2004;12:617-626.

13. Warburton PE, Dolled M, Mahmood R, et al. Molecular cytogenetic analysis of eight inversion duplications of human chromosome $13 q$ that each contain a neocentromere. Am J Hum Genet 2000;66:1794-1806.

14. Choo KH. Centromere DNA dynamics: latent centromeres and neocentromere formation. Am J Hum Genet 1997;61:1225-1233.

15. Ventura M, Mudge JM, Palumbo V, et al. Neocentromeres in 15q24-26 map to duplicons which flanked an ancestral centromere in 15q25. Genome Res 2003;13:2059-2068.

16. du Sart D, Cancilla MR, Earle E, et al. A functional neo-centromere formed through activation of a latent human centromere and consisting of nonalpha-satellite DNA. Nat Genet 1997;16:144-153.
17. Stankiewicz P, Park SS, Inoue K, Lupski JR. The evolutionary chromosome translocation 4;19 in Gorilla gorilla is associated with microduplication of the chromosome fragment syntenic to sequences surrounding the human proximal CMT1A-REP. Genome Res 2001;11:1205-1210.

18. Gratacòs M, Nadal M, Martín-Santos R, et al. A polymorphic genomic duplication on human chromosome 15 is a susceptibility factor for panic and phobic disorders. Cell 2001;106:367-379.

19. Scott SA, Cohen N, Brandt T, Warburton PE, Edelmann L. Large inverted repeats within Xp11.2 are present at the breakpoints of isodicentric X chromosomes in Turner syndrome. Hum Mol Genet 2010;19:3383-3393.

20. Chen CP, Lin CC, Li YC, et al. Clinical, cytogenetic, and molecular analyses of prenatally diagnosed mosaic tetrasomy for distal chromosome $15 q$ and review of the literature. Prenat Diagn 2004;24:767-773.

21. Spiegel M, Hickmann G, Senger G, Kozlowski P, Bartsch O. Two new cases of analphoid marker chromosomes. Am J Med Genet A 2003;116A:284289.

22. Depinet TW, Zackowski JL, Earnshaw WC, et al. Characterization of neocentromeres in marker chromosomes lacking detectable alpha-satellite DNA. Hum Mol Genet 1997;6:1195-1204.

23. Hu J, McPherson E, Surti U, Hasegawa SL, Gunawardena S, Gollin SM. Tetrasomy $15 q 25.3->$ qter resulting from an analphoid supernumerary marker chromosome in a patient with multiple anomalies and bilateral Wilms tumors. Am J Med Genet 2002;113:82-88.

24. Huang $B$, Ning $Y$, Lamb AN, et al. Identification of an unusual marker chromosome by spectral karyotyping. Am J Med Genet 1998;80:368-372.

25. Mahjoubi F, Peters GB, Malafiej P, et al. An analphoid marker chromosome inv dup(15)(q26.1qter), detected during prenatal diagnosis and characterized via chromosome microdissection. Cytogenet Genome Res 2005; 109:485-490.

26. Rowe AG, Abrams L, Qu Y, Chen E, Cotter PD. Tetrasomy 15q25->qter: cytogenetic and molecular characterization of an analphoid supernumerary marker chromosome. Am J Med Genet 2000;93:393-398.

27. Tatton-Brown K, Pilz DT, Orstavik KH, et al. $15 q$ overgrowth syndrome: a newly recognized phenotype associated with overgrowth, learning difficulties, characteristic facial appearance, renal anomalies and increased dosage of distal chromosome 15q. Am J Med Genet A 2009;149A:147154.

28. Van den Enden A, Verschraegen-Spae MR, Van Roy N, Decaluwe W, De Praeter C, Speleman F. Mosaic tetrasomy 15q25->qter in a newborn infant with multiple anomalies. Am J Med Genet 1996;63:482-485.

29. Blennow E, Bui TH, Kristoffersson $U$, et al. Swedish survey on extra structurally abnormal chromosomes in 39105 consecutive prenatal diagnoses: prevalence and characterization by fluorescence in situ hybridization. Prenat Diagn 1994;14:1019-1028.

30. Greally MT, Carey JC, Milewicz DM, et al. Shprintzen-Goldberg syndrome: a clinical analysis. Am J Med Genet 1998;76:202-212.

31. Mégarbané A, Hokayem N. Craniosynostosis and marfanoid habitus without mental retardation: report of a third case. Am J Med Genet 1998;77:170-171.

32. Tészás A, Møller RS, Kellermayer R, et al. A cryptic unbalanced translocation resulting in del 13q and dup 15q. Am J Med Genet A 2008;146A:2570-2573.

33. Zollino M, Tiziano F, Di Stefano C, Neri G. Partial duplication of the long arm of chromosome 15: confirmation of a causative role in craniosynostosis and definition of a 15q25-qter trisomy syndrome. Am J Med Genet 1999;87:391-394.

34. Price MG, Landsverk ML, Barral JM, Epstein HF. Two mammalian UNC-45 isoforms are related to distinct cytoskeletal and muscle-specific functions. J Cell Sci 2002;115(Pt 21):4013-4023.

35. Ren JG, Li Z, Sacks DB. IQGAP1 modulates activation of B-Raf. Proc Nat/ Acad Sci USA 2007;104:10465-10469.

36. Dubois CM, Laprise MH, Blanchette F, Gentry LE, Leduc R. Processing of transforming growth factor beta 1 precursor by human furin convertase. J Biol Chem 1995;270:10618-10624.

37. Okubo $\mathrm{Y}$, Siddle $\mathrm{K}$, Firth $\mathrm{H}$, et al. Cell proliferation activities on skin fibroblasts from a short child with absence of one copy of the type 1 insulin-like growth factor receptor (IGF1R) gene and a tall child with three copies of the IGF1R gene. J Clin Endocrinol Metab 2003;88: 5981-5988.

38. Cunningham ML, Horst JA, Rieder MJ, et al. IGF1R variants associated with isolated single suture craniosynostosis. Am J Med Genet A 2011;155A:91-97. 